\title{
Reentry and Rotors
}

\author{
A Rabinovitch ${ }^{1 *}$, I Aviram¹, Y Biton ${ }^{1}$ and D Braunstein ${ }^{2}$ \\ ${ }^{1}$ Physics Department, Ben-Gurion University of the Negev, Beer-Sheva, 84105, Israel \\ ${ }^{2}$ Physics Dept. Sami Shamoon College of Engineering, Beer-Sheva, Israel
}

*Corresponding author: A Rabinovitch, Physics Department, Ben-Gurion University of the Negev, Beer-Sheva, 84105, Israel

To Cite This Article: A Rabinovitch*, I Aviram, Y Biton, D Braunstein. Reentry and Rotors. Am J Biomed Sci \& Res. 2019 - 1(4). AJBSR.MS.ID.000535. DOI: 10.34297/AJBSR.2019.01.000535

Received: February 22, 2019 | Published: February 26, 2019

\section{Introduction}

Two interesting papers [1,2] appeared recently discussing the important question of a rotor (spiral) induced by a reentry mechanism and its influence on heart failures. One of the main malfunctions of the heart is tachycardia $[3,4]$, rapid heartbeat, either in the atria termed AT or in the ventricles (VT). Tachycardia can lead to fibrillation [5]. (AF or VF respectively), i.e. rather chaotic heart-waves. It is known that patients suffering from fibrosis are more prone to contract such fibrillary condition, which can lead even to sudden cardiac death (SCD). These activities are thought by most authors to be sustained by a small number of self-organized, rapidly spinning electrical rotors [6]. The importance of locating the rotor centers for ablation purposes in AF patients was shown [7] to be of real benefit in this treatment. The rotor itself can be due to a "reentry" [8], i.e. a mechanism where an action potential (AP) pulse is repeatedly oscillating around a specific route of cardiomyocytes. Actually, the term "reentry" is used in the literature both in the above restricted meaning and also to refer to the overall occurrence moving around a center such as the rotor.

Most existing papers trying to understand the development and influence of rotors in the heart use models which include fibrosis, and introduce the rotors artificially [9]. Recent articles try to understand the arrhythmias developed by the encounter of AP wave fronts and fibrotic tissues [10]. However, the question of the transformation of an AP pulse rotating around a reentry route into a spiral was not addressed.

The two papers mentioned above try to challenge the spiral creation problem. But, although both papers use mathematical models to support their points, their emphasis is different. While Balaban et al's model describes a fibrosis stricken tissue and derives the conditions under which a reentry mechanism, including a rotor, is induced there, Rabinovitch et al's model deals with the theoretical question of how a reentry route transforms into a spiral.

Balaban et al's model is based on real data of heart tissue images. It distinguishes between three types of "reentries", micro-, macro- reentries (reentry routes) and rotors and concentrates on the tissue fibrosis situation to asses when each type would appear. In order to do so, it divides the fibrotic stress into two different types, interstitial and replacement and the tissue is simulated by a mesh of triangular cells.

Interstitial fibrosis is represented by changing the edges of tissue cells rendering them electrically non-conducting thus creating a no-flux boundary there. Replacement fibrosis is carried out by removing cells from the mesh rendering the space there electrically non-conducting.

The main new result is that rotors initiate almost exclusively in interstitial fibrosis and are very scarce in the replacement one and, as expected, appearance of all "reentry" types is enhanced in conditions of reduced electrical conductivity of the heart.

Rotor formation was observed "when relatively large wave fronts pivoted and folded back onto an area of transient conduction block". This description is rather loose and may be connected to the detailed explanation of the core encounter of Rabinovitch et al (see below).

Rabinovitch et al. [11] model, based on the Morris Lecar system of equations, considers a theoretical ring (annulus) around which a single pulse of AP is rotating. The ring is surrounded by nonconducting region simulating fibrotic heart syncytium. At a certain time and position along the ring, the pulse becomes free to enter either the outside part and/or the inner part of the annulus. Results show that, in this case, partial target waves (PTW) are originated and there are basically two methods by which spirals can be originated, both depend on encounters of PTW arms; one being an encounter of an arm's end with the core and the other is a mutual encounter of two different arms. In both encounters, a partial annihilation occurs at the contact.

The Core encounter (Figure 1) is the new result of this paper. The PTW has two arms, both skirting the inner core in opposite 
directions. Approach of the distal arm to the core is hindered at first due to the refractory period of the original pulse. When this refractory region terminates, this tip encounters the core and continues to move around it. This encounter gives the arm's end a slight rotation since its part that is close to the core moves slower than the outside arm. This end breaks down into two entities, an outside arm, which continues the former movement around the core, and a "portion" that develops into a single rotor.

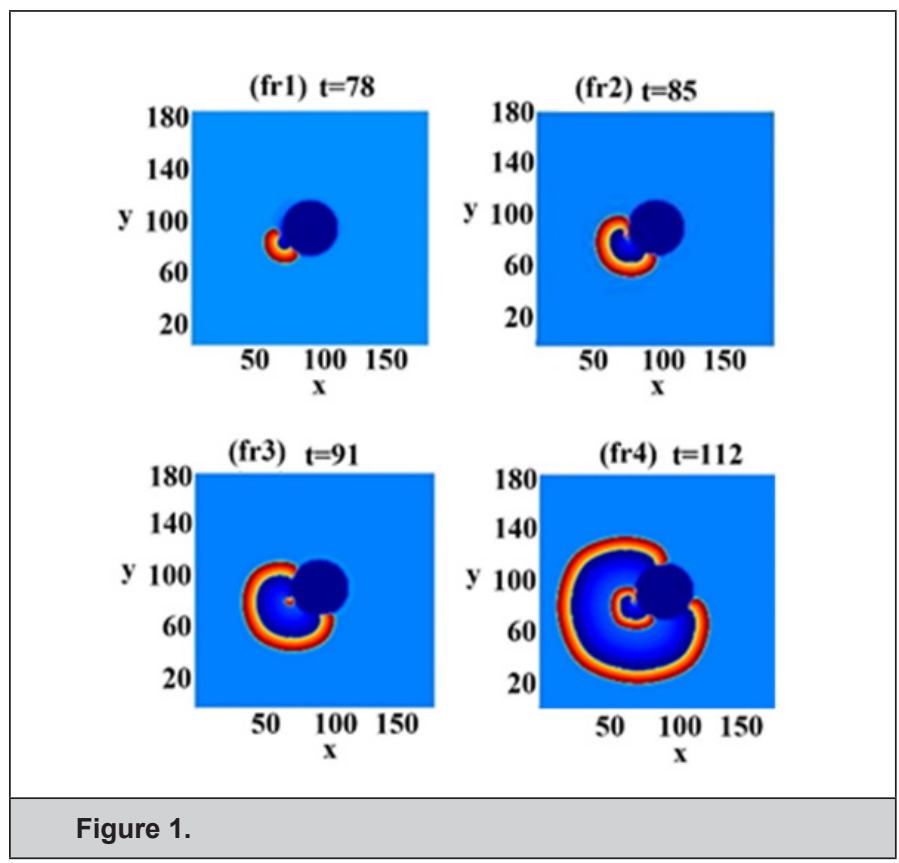

The Arms encounter has already been shown before [11]. When two arms of a PTW meet, their encounter always leads to their mutual annihilation, and the now connected arms complete a closed wave, spreading out as a target wave. However, if the arms are twisted backwards enough, the encounter can, besides creating the target wave, also lead to a "portion", this time a germ of a new double spiral. Although based on a simple mathematical model (the Morris-Lecar system), the basic understanding of how a reentry route can develop into malevolent spirals is both novel and interesting.

\section{References}

1. Balaban G, Halliday BP, Costa CM, Bai W, Porter B, et al. (2018) Fibrosis microstructure modulates reentry in non-ischemic dilated cardiomyopathy: insight from imaged 2D computational modeling, Frontiers in Physiol 19(9): 1832.

2. Rabinovitch A, Aviram I, Biton Y, Braunstein D (2018) Reentry as an Origin for Rotors Bull Math Biol 80(11): 3023-3027.

3. Brugada J, Boersma L, Allessie M, Navarro-Lopez F (1993) The complexity of mechanisms in ventricular tachycardia Pacing and clinical electrophysiology: PACE 16: 680-686.

4. Kotech D, Piccini JP (2015) Atrial fibrillation in heart failure: what should we do? Europ. Heart J 36(46): 3250-3257.

5. Schotten U, Dobrev D, Platonov PG, Kottkamp H, Hindricks G (2016) Current controversies in determining the main mechanisms of atrial fibrillation J Intern Med 279(5): 428-438.

6. Berenfeld O, Jalife J (2016) Mechanisms of Atrial Fibrillation: Rotors, Ionic Determinants, and Excitation Frequency Heart failure clinics 12: 167-178.

7. Seitz J, Bars C, Théodore G, Beurtheret S, Lellouche N, et al. (2017) AF Ablation Guided by Spatiotemporal Electrogram Dispersion Without Pulmonary Vein Isolation: A Wholly Patient-Tailored Approach Journal of the American College of Cardiology 69(3): 303-321.

8. Di Diego JM, Antzelevitch C (2014) Acute myocardial ischemia: cellular mechanisms underlying ST segment elevation J Electrocardiol 47(4): 486-490.

9. Vandersickel $\mathrm{N}$, Watanabe $\mathrm{M}$, Tao $\mathrm{Q}$ fostier J, Zeppenfeld $\mathrm{K}$, et al. (2018) Dynamical anchoring of distant arrhythmia sources by fibrotic regions via restructuring of the activation pattern. PLOS Comp Biol 14: e1006637.

10. Roney CH, Bayer JD, Zahid S, Meo M, Boyle PM, Trayanova NA, et al. (2016) Modelling methodology of atrial fibrosis affects rotor dynamics and electrograms. EPEuropace18 (4): iv146-iv155.

11. Biton Y, Rabinovitch A, Aviram I, Braunstein D (2009) Two mechanisms of spiral-pair-source creation in excitable media Phys. Lett A 373: 17621767. 